\title{
CIENCIAMATRIA
}

Revista Interdisciplinaria de Humanidades, Educación, Ciencia y Tecnología

Año VIII. Vol. VIII. Nro 1. Edición Especial. 2022

Hecho el depósito de ley: pp201602FA4721

ISSN-L: 2542-3029; ISSN: 2610-802X

Universidad Nacional Experimental Francisco de Miranda (UNEFM). Santa Ana de Coro. Venezuela

Damian Enrique Habid-Barrera; Luis Antonio Rivera-Velasco; Manacés Esaud Gaspar-Santos

DOI $10.35381 / \mathrm{cm} \cdot \mathrm{v} 8 \mathrm{i} 1.660$

\section{Responsabilidad jurídica por daño ambiental producto de la explotación minera}

\section{Legal liability for environmental damage resulting from mining}

\author{
Damian Enrique Habid-Barrera \\ dq.damianehb49@uniandes.edu.ec \\ Universidad Regional Autónoma de los Andes, Quevedo \\ Ecuador \\ https://orcid.org/0000-0002-3320-8946 \\ Luis Antonio Rivera-Velasco \\ uq.luisrivera@uniandes.edu.ec \\ Universidad Regional Autónoma de los Andes, Quevedo \\ https://orcid.org/0000-0003-2094-9566 \\ Ecuador \\ Manacés Esaud Gaspar-Santos \\ uq.manacesgaspar@uniandes.edu.ec \\ Universidad Regional Autónoma de los Andes, Quevedo \\ https://orcid.org/0000-0003-4929-4495 \\ Ecuador
}

Recibido: 15 de septiembre 2021

Revisado: 10 de noviembre 2021

Aprobado: 15 de diciembre 2021

Publicado: 01 de enero de 2022 
CIENCIAMATRIA

Revista Interdisciplinaria de Humanidades, Educación, Ciencia y Tecnología

Año VIII. Vol. VIII. Nro 1. Edición Especial. 2022

Hecho el depósito de ley: pp201602FA4721

ISSN-L: 2542-3029; ISSN: 2610-802X

Universidad Nacional Experimental Francisco de Miranda (UNEFM). Santa Ana de Coro. Venezuela

Damian Enrique Habid-Barrera; Luis Antonio Rivera-Velasco; Manacés Esaud Gaspar-Santos

\title{
RESUMEN
}

El objetivo general de la investigación fue analizar la responsabilidad jurídica por daño ambiental producto de la explotación minera. En el desarrollo de este artículo se aplicó la modalidad cuantitativa, teniendo presente la especificación del objeto de estudio. Apoyado en un diseño bibliográfico-documental, indagación que posibilita tener una explicación amplia del tema posibilitando comprender una extensa gama de fenómeno, con la revisión de artículos arbitrados, leyes y normas relacionados al tema. Se necesita reconocer que las instituciones juegan un papel fundamental en la gestión ambiental. Se concluye que en el Ecuador, las herramientas legales para sancionar y asegurar la relación de los males del medio ambiente, no son aplicados de forma inexorable y constante de parte de las autoridades del medio ambiente; ocasionando de alguna forma solamente el incremento de delincuentes ecológicos en la mala práctica de la minería, al punto de ocasionar nefastas secuelas para el medio ambiente nacional.

Descriptores: Responsabilidad civil; medio ambiente; minería. (Tesauro UNESCO).

\begin{abstract}
The general objective of the research was to analyze the legal liability for environmental damage resulting from mining exploitation. In the development of this article, the quantitative modality was applied, bearing in mind the specification of the object of study. Supported by a bibliographic-documentary design, an inquiry that makes it possible to have a broad explanation of the subject, making it possible to understand a wide range of phenomena, with the review of peer-reviewed articles, laws and regulations related to the subject. It is necessary to recognize that institutions play a fundamental role in environmental management. It is concluded that in Ecuador, the legal tools to sanction and assure the relation of the evils of the environment, are not applied in an inexorable and constant way on the part of the authorities of the environment; causing in some way only the increase of ecological delinquents in the bad practice of mining, to the point of causing harmful consequences for the national environment.
\end{abstract}

Descriptors: Liability; environment; mining. (UNESCO Thesaurus). 


\section{CIENCIAMATRIA}

Revista Interdisciplinaria de Humanidades, Educación, Ciencia y Tecnología

Año VIII. Vol. VIII. Nro 1. Edición Especial. 2022

Hecho el depósito de ley: pp201602FA4721

ISSN-L: 2542-3029; ISSN: 2610-802X

Universidad Nacional Experimental Francisco de Miranda (UNEFM). Santa Ana de Coro. Venezuela

Damian Enrique Habid-Barrera; Luis Antonio Rivera-Velasco; Manacés Esaud Gaspar-Santos

\section{INTRODUCCIÓN}

Diversos principios jurídicos y puntos de vista de derecho ambiental que enuncia la Constitución de Montecristi ya habían sido establecidos por la Constitución de 1998, no obstante quisiera probar aquí preguntas que no permanecen reguladas por la Constitución de 1998 y que, junto con otras innovaciones de la nueva abreviatura, como por ejemplo los derechos de la naturaleza, el derecho humano al agua, el principio de la prevalencia a favor de la naturaleza, el principio de la restauración integral, el principio de la solidaridad, la subsidiariedad del Estado y la imprescriptibilidad de las actividades del medio ambiente, entre otros, que merecen ser analizados en capítulo aparte, traerán secuelas positivas para la justicia ambiental en el territorio. (Crespo-Plaza, 2008, p.1)

Al respecto, se relata el compromiso objetivo por mal ambiental y al reconocimiento de la carga de la prueba; estas dos innovaciones de la Carta Magna ecuatoriana podrían mejorar el derecho a la justicia ambiental, en el sentido de que el Estado como individuos no está obligado a reconocer los costos fundamentales que integran la prueba del mal ambiental al tergiversar esta obligación criminal hacia la tergiversación.

Por lo tanto, la impunidad ambiental en el territorio se mantiene en un grado extremo debido a que los costos de las pruebas, peritajes y la dificultad de revisar la causa efecto en los temas del medio ambiente masculinos se transforman en una barrera para el ingreso de la justicia ambiental, tomando en cuenta que por lo general las víctimas son personas con discapacidad de recursos económicos. El mal ambiental es una manera directa vinculada a la salud pública, es fundamental el progreso que se ha tenido tanto a grado nacional como mundial en este asunto. La prevención es un hecho necesario para asegurar un ámbito sana que garantice un mejor estilo de vida.

En este sentido, la responsabilidad ambiental tiene lugar para resarcir a los sujetos resultantes del resultado de un desenlace mal provocativo para el medio ambiente, mediante la reposición parcial o total del área afectada. En cuanto el transgresor se vea obligado a reparar el daño causado, deberá modificar o cesar ocupaciones lesivas al bien 


\section{CIENCIAMATRIA \\ Revista Interdisciplinaria de Humanidades, Educación, Ciencia y Tecnología \\ Año VIII. Vol. VIII. Nro 1. Edición Especial. 2022 \\ Hecho el depósito de ley: pp201602FA4721 \\ ISSN-L: 2542-3029; ISSN: 2610-802X \\ Universidad Nacional Experimental Francisco de Miranda (UNEFM). Santa Ana de Coro. Venezuela}

Damian Enrique Habid-Barrera; Luis Antonio Rivera-Velasco; Manacés Esaud Gaspar-Santos

común, hasta el punto de que los costos por utilizar tecnologías y sistemas para minimizar los contaminantes sean inferiores a los costos que tiene que cubrir como consecuencia de las sanciones impuestas por la superación de las perturbaciones previstas por la legislación aplicable.

Sin embargo, los inconvenientes del medio ambiente son recurrentes en cada una de las metrópolis del Ecuador, por lo cual es efectivo hacer un análisis referente a cómo deteriora la explotación minera en el medio ambiente y saber cómo prevenirlo por medio de la aplicación positiva de la ley y el interés, responsabilidad y prioridad que se debería auxiliar el sistema sobre los padecimientos del medio ambiente, generados por equivocados mecanismos innovadores que contribuyen a la colaboración para la custodia de la vida.

Al respecto, la Constitución de la República del Ecuador(2008) consagra que todos los ecuatorianos poseemos derecho a vivir en un ambiente sano que garantice nuestro buen vivir, es por esto que si se aplicara de manera correcta la ley en temas de defensa ambiental, los beneficiarios podrían ser todos esos que habitan en este territorio rico en zonas verdes y enorme variedad de flora y fauna que nos hace envidiar a muchas naciones de todo el mundo, que todavía disfrutan del privilegio de respirar viento puro en ciertos sitios del Ecuador.

La responsabilidad penal por la minería ilegal en el flujo de agua Quevedo tiene enorme relevancia jurídica y social, debido a que este suceso produce violaciones a las leyes vigentes que la regulan; por lo cual la sustracción ilegal es una realidad en el Ecuador, y que en los últimos años ha ido en crecimiento y dejando grandes antecedentes tanto en la naturalidad como en la convivencia de los seres vivos con la Pachamama.

En la actualidad, el mal ambiental clasifica como patrimonial y extrapatrimonial. La primera es la que recae sobre bienes posibles de costo económico, sean dichos corporales o incorporales, o los que no tengan carácter patrimonial como la vida, la salud; Por otro lado, el mal de tipo extrapatrimonial o moral es ese que no conduce a una 


\section{CIENCIAMATRIA \\ Revista Interdisciplinaria de Humanidades, Educación, Ciencia y Tecnología \\ Año VIII. Vol. VIII. Nro 1. Edición Especial. 2022 \\ Hecho el depósito de ley: pp201602FA4721 \\ ISSN-L: 2542-3029; ISSN: 2610-802X \\ Universidad Nacional Experimental Francisco de Miranda (UNEFM). Santa Ana de Coro. Venezuela}

Damian Enrique Habid-Barrera; Luis Antonio Rivera-Velasco; Manacés Esaud Gaspar-Santos

disminución de los activos recurriendo a bienes principales que no poseen probabilidad de ser valorados por una perspectiva pecuniaria, cuya forma única de compostura se inspira no obstante sobre la compensación económica, desde la cual se incorporan las heridas con los derechos de la personalidad, con los derechos primordiales, particulares o colectivos, así como con los sufrimientos y molestias derivados de tales heridas. (PeñaChacón, 2020, p. 6)

Así mismo, no existe una preocupación por el problema ambiental, lo que ha llevado a examinar la gravedad de los desequilibrios en el medio ambiente, a raíz del aumento de la población y de las ocupaciones humanas, que han causado daños a los recursos renovables, sin embargo, además, estos seguirán sin los recursos renovables se han agotado. El interés que despierta la protección del medio ambiente gira en torno a la salud y la paz de las personas, por lo que deben tener una reacción consciente de que somos parte de este mundo, que es nuestro hogar exclusivo. (Castillo-Osorio, 2018, p.2)

El sistema de responsabilidad conserva como punto central la obligación de resarcir el mal, por lo cual, en caso de mal, debería entenderse como el factor que constituye la columna vertebral de todo sistema de responsabilidad. Es por esto que para usar cualquier sistema de responsabilidad (civil, administrativa, penal o ambiental), se necesita antes que nada probar la vida de un infortunio. Esta concepción del mal ha evolucionado $y$ hoy es viable que exista el mal, aunque no recaiga justamente en los derechos privados o reales, como es la situación del puro mal ecológico.

La indemnización no posee sentido en la compostura del mal hacia el medio ambiente, debido a que con ella no se repara el objeto dañado, hasta que un sustituto pecuniario logre regresar íntegramente el bien ambiental. Para eso, se necesita buscar constantemente herramientas eficaces para la evaluación del mal y su compostura. En impacto, sin perjuicio de los mandatos impuestos por los inicios del derecho ambiental, en especial los inicios de prevención, precaución y quien contamina paga, nos parece que atribuir responsabilidad a un individuo en esas situaciones en las que era imposible 


\section{CIENCIAMATRIA}

Revista Interdisciplinaria de Humanidades, Educación, Ciencia y Tecnología

Año VIII. Vol. VIII. Nro 1. Edición Especial. 2022

Hecho el depósito de ley: pp201602FA4721

ISSN-L: 2542-3029; ISSN: 2610-802X

Universidad Nacional Experimental Francisco de Miranda (UNEFM). Santa Ana de Coro. Venezuela

Damian Enrique Habid-Barrera; Luis Antonio Rivera-Velasco; Manacés Esaud Gaspar-Santos

pronosticar el mal, implicaría la producción de una situación de inseguridad jurídica contraria a la ley que vulneraría otros principios que inspiran el derecho ambiental, a saber, el del desarrollo sustentable.

Por lo tanto, la responsabilidad administrativa ambiental es la que se deriva de la violación de la norma administrativa ambiental, de sus normas y reglamentos complementarios, si se especifica en la imposición de la sanción administrativa por la violación u omisión, y de ella deriva la obligación de resolver la agresión, de utilizar medidas de prevención y mitigación y hacerse cargo de los costos correspondientes.

El Estado, a través de los múltiples niveles del régimen, tiene la obligación de hacer valer los derechos consagrados en la Constitución, para lo cual realiza actividades políticas que conducen a la protección de los derechos establecidos por ella. Siendo el derecho a un ambiente sano uno de los derechos tutelares del Estado, debe generarse a través de los múltiples niveles del sistema de políticas públicas que establecen competencias, manejo de recursos, sistemas de prevención, evaluación y control ambiental, etc., para proteger este bien jurídico.

Estas ocupaciones deben tener lugar en las interacciones entre el Estado y la sociedad, para lo cual deben establecerse las obligaciones de los administradores y las correspondientes infracciones y sanciones, teniendo en cuenta las oportunidades, garantías y proporciones para el logro de los objetivos de desarrollo sostenible. De estas sinergias surgen actividades administrativas, afines a la defensa, control y manejo de los recursos naturales. La regla administrativa se convierte en regla de conducta en cuanto a la gestión o conducta de los sujetos en defensa del medio ambiente y en regla organizativa al establecer las jerarquías o niveles de empleo y las relaciones entre estos niveles junto con el papel del régimen del Estado y del Territorio. Alcanzar los objetivos del derecho ambiental.

De acuerdo a los planteamientos formulados se presenta como objetivo general de la investigación analizar la responsabilidad jurídica por daño ambiental producto de la 


\section{CIENCIAMATRIA}

Revista Interdisciplinaria de Humanidades, Educación, Ciencia y Tecnología

Año VIII. Vol. VIII. Nro 1. Edición Especial. 2022

Hecho el depósito de ley: pp201602FA4721

ISSN-L: 2542-3029; ISSN: 2610-802X

Universidad Nacional Experimental Francisco de Miranda (UNEFM). Santa Ana de Coro. Venezuela

Damian Enrique Habid-Barrera; Luis Antonio Rivera-Velasco; Manacés Esaud Gaspar-Santos

explotación minera.

\section{METODOLOGÍA}

En el desarrollo de este artículo se aplicó la modalidad cuantitativa, teniendo presente la especificación del objeto de estudio. Apoyado en un diseño bibliográfico-documental, indagación que posibilita tener una explicación externa e interna personal de cada libro a fin de tener una perspectiva conjunta de todo el material informativo; el cual ha sido usado en el curso de averiguación y desarrollo del asunto propuesto. (Gómez-Luna, 2014). Así mismo, una de las primordiales ventajas de un diseño bibliográfico-documental es que posibilita comprender una extensa gama de fenómenos debido a que encierra una realidad espacio-temporal muchísimo más vasta en el asunto de averiguación postulado. Esta clase de análisis busca solamente explicar situaciones o eventos; luego de todo, no está interesado en revisar satisfactoriamente o en probar cierta conjetura, o en hacer predicciones. Constantemente las descripciones se realizan desde encuestas (survey study), aunque estas además tienen la posibilidad de servir para contrastar conjeturas específicas y colocar pruebas demostrativas. (Guevara-Albán, 2020)

\section{RESULTADOS}

Se puede deducir que el mal ambiental se confundirá con la mala causa del mal ambiental. El primero hace referencia al efecto negativo del acento antrópico en el medio ambiente; el segundo, al grupo de recursos que aparecen como las diversas secuelas del mal para la víctima del mismo. Por consiguiente, debería decidirse que para los efectos de este trabajo se plantea mal ambiental acorde a la definición anterior. Partiendo de un criterio biológico, el mal ambiental es el efecto de una acción antrópica que confiere el equilibrio homeostático, fisiológico y/o genética de un organismo vivo, intacta al propio hombre. Desafortunadamente, a las comunidades o no se les informa acerca de los proyectos mineros o se les informa mal o los intereses económicos juegan un papel clave 


\section{CIENCIAMATRIA \\ Revista Interdisciplinaria de Humanidades, Educación, Ciencia y Tecnología \\ Año VIII. Vol. VIII. Nro 1. Edición Especial. 2022 \\ Hecho el depósito de ley: pp201602FA4721 \\ ISSN-L: 2542-3029; ISSN: 2610-802X \\ Universidad Nacional Experimental Francisco de Miranda (UNEFM). Santa Ana de Coro. Venezuela}

Damian Enrique Habid-Barrera; Luis Antonio Rivera-Velasco; Manacés Esaud Gaspar-Santos

(La Rotta-Latorre x6y Torres-Tovar, 2017, p.78).

Es importante señalar los criterios que nos permiten creer que un comportamiento merece una sanción penal y no sencillamente una sanción administrativa. Al respecto, hay dos posiciones en el ideario: la primera se fundamenta en la comparación del comportamiento constitutiva de infracción administrativa con el comportamiento constitutivo de infracción penal. Cualquier infracción de esta clase se convierte en un delito potencial.

La segunda postura se fundamenta en sancionar solamente las conductas que dañen gravemente los recursos naturales, apreciándose un componente especialmente cuantitativo, referido a la cuantía de la carga del mal sobre el recurso natural. Esta postura se fundamenta en la valoración de los delitos ecológicos como delitos de peligro concreto, en los cuales no basta la ejecución de un comportamiento prohibida por la ley, sino que la vida de un componente adicional es sustancialmente elemental: En el caso concreta, el juez establece si dicha situación peligrosa prohibida por la tipología delictiva es positiva y verdaderamente ha desarrollado una regla estricta para el bien jurídico, o si en realidad ha dañado gravemente el recurso natural o el medio ambiente. Al respecto, VásconezCarrasco y Torres-León (2018) indican:

En el caso ecuatoriano, la Constitución delimita la actividad minera requiriendo especialmente la sostenibilidad. En general, podemos encontrar artículos que reconocen a la población la garantía de la sostenibilidad (Art. 14); la producción en general deberá guiarse por principios de sostenibilidad (Art. 320); y lo propio sucede en el caso de la seguridad social (Art. 368). Las actividades que atenten contra los derechos de la naturaleza serán desincentivadas por parte del Estado (Art. 319). (p.86)

El sistema de rendición de cuentas mantiene como punto central la obligación de reparar el dolor, por tanto, si se trata del dolor como elemento constitutivo de la columna vertebral del sistema de rendición de cuentas. Es por ello que para ejercer cualquier sistema de responsabilidad (civil, administrativa, penal o ambiental) si es necesario probar la 


\section{CIENCIAMATRIA \\ Revista Interdisciplinaria de Humanidades, Educación, Ciencia y Tecnología \\ Año VIII. Vol. VIII. Nro 1. Edición Especial. 2022 \\ Hecho el depósito de ley: pp201602FA4721 \\ ISSN-L: 2542-3029; ISSN: 2610-802X \\ Universidad Nacional Experimental Francisco de Miranda (UNEFM). Santa Ana de Coro. Venezuela}

Damian Enrique Habid-Barrera; Luis Antonio Rivera-Velasco; Manacés Esaud Gaspar-Santos

autenticidad de un agravio. Esta concepción del mal ha evolucionado y hoy es posible que el mal exista, aunque obviamente no recaiga sobre derechos individuales o reales, como en la situación de pura enfermedad ecológica. La indemnización no tiene sentido en la reparación del daño al medio ambiente, porque con ella no se repara el objeto dañado, hasta que un sustituto pecuniario no puede devolver íntegramente el bien ambiental. Los autores Massa-Sánchez, Cisne-Arcos y Maldonado (2018) formulan lo siguiente:

El análisis de los recursos agotables, su explotación y relación con la interacción social implica establecer que la sostenibilidad fuerte debe primar para garantizar la generación de recursos que inicialmente beneficien y minimicen los cambios que necesariamente influirán en las personas y la vida silvestre comprometidas. (p.125)

Por lo tanto, toda persona, comunidad, pueblo o nacionalidad podrá exigir a la autoridad el cumplimiento de los derechos de la naturaleza (Gregor, 2014).

\section{DISCUSIÓN}

Se necesita reconocer que las instituciones juegan un papel fundamental en la gestión económica de las naciones y, sobre todo, en la funcionalidad distributiva de los recursos patrimoniales, pues ponen de relieve las reglas que guían la actividad humana en una sociedad. Los individuos y las empresas invierten permanentemente en seguros solo para obtener ganancias, independientemente de las repercusiones sociales y del medio ambiente, y que la función de la organización para imponerse a la autoridad del territorio es fundamental.

El derecho a un medio ambiente sano es el derecho de todo individuo, en equidad de condiciones, a vivir en un lugar benefactor que le proporcione recursos naturales, artificiales, para hacer realidad su realidad y su desarrollo, así como como otros organismos vivos interactuando en un espacio y tiempo determinado.

En la práctica, es una de las actividades mineras ilegales que perjudican el medio 


\section{CIENCIAMATRIA}

Revista Interdisciplinaria de Humanidades, Educación, Ciencia y Tecnología

Año VIII. Vol. VIII. Nro 1. Edición Especial. 2022

Hecho el depósito de ley: pp201602FA4721

ISSN-L: 2542-3029; ISSN: 2610-802X

Universidad Nacional Experimental Francisco de Miranda (UNEFM). Santa Ana de Coro. Venezuela

Damian Enrique Habid-Barrera; Luis Antonio Rivera-Velasco; Manacés Esaud Gaspar-Santos

ambiente, en especial la contaminación de suelos y aguas, superando los niveles permitidos por la legislación ambiental.

\section{CONCLUSIÓN}

En el Ecuador, las herramientas legales para sancionar y asegurar la relación de los males del medio ambiente, no son aplicados de forma inexorable y constante de parte de las autoridades del medio ambiente; ocasionando de alguna forma solamente el incremento de delincuentes ecológicos en la mala práctica de la minería, al punto de ocasionar nefastas secuelas para el medio ambiente.

\section{FINANCIAMIENTO}

No Monetario.

\section{AGRADECIMIENTO}

A la Universidad Regional Autónoma de los Andes, Quevedo; por motivar el desarrollo de la investigación.

\section{REFERENCIAS CONSULTADAS}

$\mathrm{X} \mid$

(C2022 por los autores. Este artículo es de acceso abierto y distribuido según los términos y condiciones de la licencia Creative Commons Atribución-NoComercial-Compartirlgual 4.0 Internacional (CC BY-NC-SA 4.0)

(https://creativecommons.org/licenses/by-nc-sa/4.0/). 


\section{CIENCIAMATRIA}

Revista Interdisciplinaria de Humanidades, Educación, Ciencia y Tecnología

Año VIII. Vol. VIII. Nro 1. Edición Especial. 2022

Hecho el depósito de ley: pp201602FA4721

ISSN-L: 2542-3029; ISSN: 2610-802X

Universidad Nacional Experimental Francisco de Miranda (UNEFM). Santa Ana de Coro. Venezuela

Damian Enrique Habid-Barrera; Luis Antonio Rivera-Velasco; Manacés Esaud Gaspar-Santos 